\title{
Impact of device geometry on electron and phonon transport in graphene nanorings
}

\author{
M. Saiz-Bretín,,${ }^{1,2}$ L. Medrano Sandonas, ${ }^{2,3}$ R. Gutierrez, ${ }^{2}$ G. Cuniberti, ${ }^{2,3,4}$ and F. Domínguez-Adame ${ }^{1}$ \\ ${ }^{1}$ GISC, Departamento de Física de Materiales, Universidad Complutense, E-28040 Madrid, Spain \\ ${ }^{2}$ Institute for Materials Science, TU Dresden, 01062 Dresden, Germany \\ ${ }^{3}$ Center for Advancing Electronics Dresden, TU Dresden, 01062 Dresden, Germany \\ ${ }^{4}$ Dresden Center for Computational Materials Science, TU Dresden, 01062 Dresden, Germany
}

(Received 12 February 2019; published 26 April 2019)

\begin{abstract}
Recent progress in nanostructuring of materials opens up possibilities to achieve more efficient thermoelectric devices. Nanofilms, nanowires, and nanorings may show increased phonon scattering while keeping good electron transport, two of the basic ingredients for designing more efficient thermoelectric systems. Here we argue that graphene nanorings attached to two leads meet these two requirements. Using a density-functional parametrized tight-binding method combined with Green's function technique, we show that the lattice thermal conductance is largely reduced as compared to that of graphene nanoribbons. At the same time, numerical calculations based on the quantum transmission boundary method, combined with an effective transfer matrix method, predict that the electric properties are not considerably deteriorated, leading to an overall remarkable thermoelectric efficiency. We conclude that graphene nanorings can be regarded as promising candidates for nanoscale thermoelectric devices.
\end{abstract}

DOI: 10.1103/PhysRevB.99.165428

\section{INTRODUCTION}

Exploration of new materials and novel physical mechanisms for heat-to-electricity conversion lies at the heart of thermoelectricity research. The thermoelectric efficiency of materials and devices can be quantified by the dimensionless figure of merit $Z T=\sigma S^{2} T / \kappa$ [1]. Here $S$ stands for the Seebeck coefficient, and $\sigma$ and $\kappa$ are the electrical and thermal conductance at temperature $T$, respectively. Although the figure of merit has formally no upper bound, values of $Z T$ larger than 3 are usually considered as necessary for widespread applications. Bulk materials are, however, usually well below this threshold. One obvious strategy for improving $Z T$ is to reduce the thermal conductivity $\kappa$, while maximizing the power factor $\sigma S^{2}$. However, the unfavorable interdependence of $S, \sigma$, and $\kappa$ in three-dimensional (3D) systems is the main cause of the low efficiency of current thermoelectric devices compared to other energy-conversion technologies. In general, both electrons and phonons can contribute to the heat current through a system subjected to a temperature gradient. Thus, the thermal conductance is split as $\kappa=\kappa_{\mathrm{el}}+\kappa_{\mathrm{ph}}$. In this regard, reducing the phonon contribution $\kappa_{\mathrm{ph}}$ by increasing phonon scattering is an advantageous strategy to enhance $Z T$. This approach might be especially relevant when the electron density is low, as occurs in semiconductors and insulators, because the electron contribution $\kappa_{\mathrm{el}}$ is expected to be small.

It is frequently believed that phonon mean free paths $\ell_{\mathrm{ph}}$ are much longer than those of electrons $\ell_{\mathrm{el}}$. Miniaturization of materials was predicted long ago to exploit this difference in order to achieve a significant reduction of the phonon thermal conductivity while maintaining good electric properties $[2,3]$, provided that their size is larger than $\ell_{\mathrm{el}}$ but smaller than $\ell_{\mathrm{ph}}$. Furthermore, quantum effects allow nanodevices to overcome the limitations stemming from the classical Wiedemann-Franz law. For instance, sharp resonances in the electron transmission (such as Fano line shapes) make the ratio $\sigma / \kappa_{\mathrm{el}}$ increase well above the Wiedemann-Franz limit [4-11]. The combination of these two factors, namely reduced $\ell_{\mathrm{ph}}$ and enhanced $\sigma / \kappa_{\mathrm{el}}$, explains why nanometer-sized objects may exhibit values of $Z T$ unachievable with bulk materials [12-17].

Graphene is a paradigmatic example of a two-dimensional material whose thermoelectric efficiency can be significantly improved after nanostructuring or functionalization. Graphene nanoribbons with rough edges [18], hydrogen passivation [19], patterning [20-23], and hybrid graphene/hexaboron nitride superlattices [24] display a substantial reduction of the lattice thermal conductivity. The insertion of bends, corners, and other structural modifications in the phonon paths yields even lower thermal conductivity. For instance, arrays of nanopores [25] and double bends [26] drastically reduce the phonon contribution $\kappa_{\mathrm{ph}}$ in graphene nanoribbons. The reduction of the thermal conductivity after introducing obstacles, however, does not always improve thermoelectric efficiency since electrical conduction can be deteriorated as well. This has been already confirmed in polycrystalline heterostructures combining graphene and hexaboron nitride [27]. Molecular dynamics simulations show that decreasing the width, changing the termination of nanoribbons from zigzag to armchair, and roughening the edges lead to a dramatic reduction of the thermal conductivity due to an increase in the ratio of edge length to surface area [28]. Hybrid nanostructures based on graphene provide another route towards enhanced thermoelectric response. The power factor $\sigma S^{2}$ of composite films comprised of graphene quantum dots and conducting polymers was found to be $550 \%$ higher than that of the pristine polymer due to the strong interfacial interaction between the dot and the polymer chains [29]. Vertical graphene- $\mathrm{C}_{60}$-graphene architectures are scalable and 
might be used to boost thermoelectric performance [30]. Moreover, three-terminal triangular graphene nanojunctions display improved efficiency at maximum output compared to two-terminal setups [31].

Graphene nanorings occupy a unique place among graphene nanostructures in terms of their thermoelectric properties [32]. They can take advantage of quantum interference effects because the phase coherence length of electronic states in graphene is large even at room temperature [33]. These electronic effects can be exploited for designing new quantum interferometers [34-37] or spintronic devices [38-40] that are relevant to heat-to-electricity conversion as well. However, in Ref. [32] the contribution of the atomic lattice to heat transport was neglected, thus posing a question about the validity of the conclusions in actual systems since the contribution is expected to be high in graphene-based systems. In this context, nonequilibrium molecular dynamics simulations show that the lattice contribution to the thermal conductivity in graphene nanorings is only $50 \%$ of that of graphene nanoribbons of the same width [41]. It should be noticed that nonequilibrium molecular dynamics simulations exclude quantum effects and, consequently, they cannot be regarded as a valid tool for studying graphene nanostructures below $107 \mathrm{~K}$ (see Ref. [41] and references therein). Consequently, in this work we first explore the phonon transport properties in the low temperature regime by using a density-functional based tightbinding (DFTB) method [42] combined with Green's function techniques. We study three different configurations of graphene nanorings to elucidate the optimal geometry yielding the lower $\kappa_{\mathrm{ph}}$ value. Once this magnitude is determined, we then compute electron wave functions and transmission coefficients using the quantum transmission boundary method $[43,44]$ combined with the effective transfer matrix method [45]. Finally, the corresponding $Z T$ is obtained over a wide range of parameters, finding remarkable enhancement as compared to graphene nanoribbons. In Sec. II we introduce the graphene nanostructures we are going to analyze in this study, together with a description of the computational methodology. In Sec. III the results for the phonon and electron transport as well as the thermoelectric response of the devices are then discussed in detail. Finally, Sec. IV summarizes our conclusions.

\section{SYSTEM, MODEL, AND METHODOLOGY}

The system under study is a graphene rectangular ring with arms of width $w$ attached to two semi-infinite graphene nanoribbons of width $w_{0}$, which act as contacts. Depending on where these contacts are placed, we obtain different configurations, which we call symmetrical, asymmetrical, and orthogonal (see Fig. 1 for reference). Throughout this work we take $w_{0}=1.23 \mathrm{~nm}, l_{0}=1.7 \mathrm{~nm}$, and we also consider the ring to be square and uniform $\left(l=3 w_{0}, w_{1} \approx w\right)$, unless otherwise stated. Geometry optimization of the graphene nanostructures was carried out by means of the DFTB approach as implemented in the DFTB + software package [42]. This method is based on density-functional theory, but also uses ideas of the semiempirical tight-binding approach, hence the combination of accuracy and numerical efficiency. Moreover, it allows dealing with systems up to 2000 atoms (a) symmetric

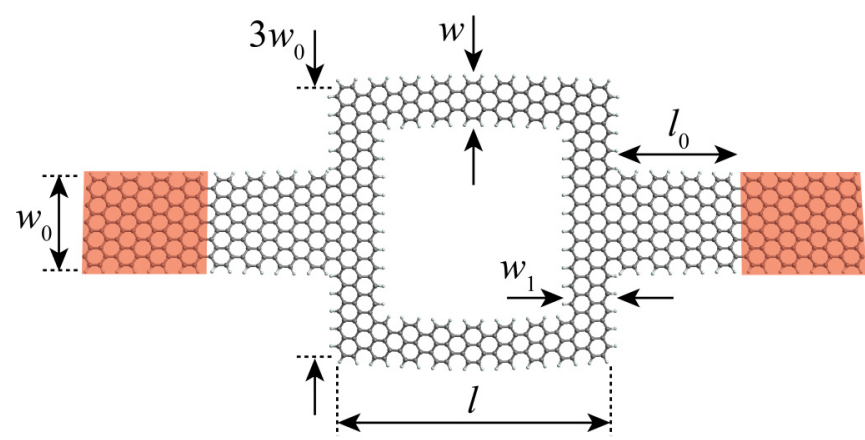

(b)

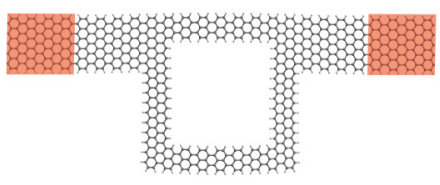

asymmetric (c)

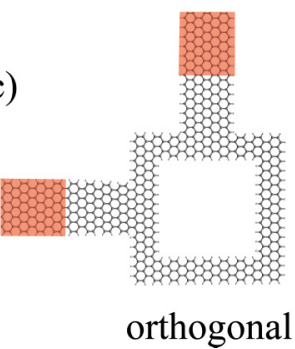

FIG. 1. Schematic diagram of the three configurations of graphene nanorings connected to two ideal leads, represented by shadowed areas. (a) Symmetrical, (b) asymmetrical, and (c) orthogonal configurations.

in a quantum simulation, especially for carbon-based nanomaterials $[46,47]$. We have used the Slater-Koster parameters obtained by Niehhaus et al. [48] for C and $\mathrm{H}$ atoms. Geometry optimization was performed by using the conjugate gradient method, until the absolute value of the interatomic forces was below $10^{-5}$ a.u.

Interestingly, most of the $\Gamma$ (including all the acoustic phonons as well) and $K$ phonon modes have been shown to be uncoupled to electrons [49]. Therefore, as a reasonable approximation, electron-phonon interaction are not be considered in our calculations. Consequently, phonon and electron transport can be treated independently within the Landauer approach in terms of the corresponding electron $\tau_{\mathrm{el}}(E)$ and phonon $\tau_{\mathrm{ph}}(\omega)$ transmission coefficients. For the low temperatures mostly considered here, phonon-phonon interactions can also be safely neglected. Phonon transport properties have been computed by combining the DFTB method with Green's function technique (GF-DFTB), as implemented in the inhouse version of DFTB + code [47,50,51]. Based on this, we divide the whole system into three regions (see Fig. 1), namely left and right contacts, and a device (scattering) region. Then we calculate the phonon transmission coefficient $\tau_{\mathrm{ph}}(\omega)$ as

$$
\tau_{\mathrm{ph}}(\omega)=\operatorname{Tr}\left(G^{r} \Gamma_{L} G^{a} \Gamma_{R}\right),
$$

where the broadening functions are $\Gamma_{L / R}=i\left(\Sigma_{L / R}^{r}-\Sigma_{L / R}^{a}\right)$ with $\Sigma_{L / R}$ being the contact self-energies. The retarded Green's function is $G^{r}=\left(\omega^{2} \mathbb{1}-K-\Sigma_{L}^{r}-\Sigma_{R}^{r}\right)^{-1}$, where $K$ is the dynamical matrix, $\omega$ is the phonon frequency, and $\mathbb{1}$ is the unit matrix. $K$ is obtained by numerically differentiating the forces calculated using the DFTB method. Hence, the 
thermal conductance is obtained as

$$
\kappa_{\mathrm{ph}}=\frac{\hbar^{2}}{2 \pi k_{B} T^{2}} \int_{0}^{\infty} \frac{\omega^{2} e^{\hbar \omega / k_{B} T}}{\left(e^{\hbar \omega / k_{B} T}-1\right)^{2}} \tau_{\mathrm{ph}}(\omega) d \omega,
$$

with $k_{B}$ and $\hbar$ as the Boltzmann and Planck constants, respectively. This expression is obtained by a linear expansion in the applied temperature difference $\Delta T$ of the quantity $N_{B}(T+$ $\Delta T)-N_{B}(T)$, where $N_{B}(T)$ is the Bose-Einstein distribution at a temperature $T$.

To calculate the electron transport properties of the graphene nanostructures, we have employed a nearestneighbor tight-binding Hamiltonian

$$
\mathcal{H}=\sum_{i} \varepsilon_{i}|i\rangle\left\langle i\left|-t \sum_{\langle i, j\rangle}\right| i\right\rangle\langle j|,
$$

where $|i\rangle$ is the $\pi$ orbital of the $i$ th carbon atom, and the hopping parameter and site energy are set to $t=2.8 \mathrm{eV}$ and $\varepsilon_{i}=0$, respectively. Assuming that electrons are in the fully coherent regime, we use the quantum transmitting boundary method $[43,44]$ combined with an effective transfer matrix method adapted for graphene [45] to calculate wave functions and the electron transmission coefficient $\tau_{\mathrm{el}}(E)$ (see Ref. [52] for further details on the calculation method). The transmission coefficient $\tau_{\mathrm{el}}(E)$ has also been calculated using GF-DFTB approach for testing purposes, obtaining an excellent agreement between both methods. Therefore, in the following we use the transfer matrix method since it demands less computational resources.

The Seebeck coefficient $S$, the electrical conductance $\sigma$, and the electronic thermal conductance $\kappa_{\mathrm{el}}$ can be calculated from the following expressions (see, e.g., Ref. [32] and references therein)

$$
\begin{aligned}
S & =-\frac{1}{e T} \frac{L_{1}}{L_{0}}, \\
\sigma & =e^{2} L_{0}, \\
\kappa_{\mathrm{el}} & =\frac{1}{T}\left(L_{2}-\frac{L_{1}^{2}}{L_{0}}\right),
\end{aligned}
$$

where the functions $L_{n}$ are given in terms of the moments of the electronic transmission function:

$$
L_{n}=\frac{2}{h} \int_{-\infty}^{\infty}\left(-\frac{\partial f}{\partial E}\right)(E-\mu)^{n} \tau_{\mathrm{el}}(E) d E .
$$

It is worth mentioning that these expressions are valid in the linear response regime. In this equation $\mu$ is the chemical potential of the graphene contacts, and $f(E)=\{\exp [(E-$ $\left.\left.\mu) / k_{B} T\right]+1\right\}^{-1}$ is the Fermi distribution function. For concreteness, we set $T=4 \mathrm{~K}$ hereafter. Finally, including the phonon thermal conductance $\kappa_{\text {ph }}$ previously calculated, the thermoelectric figure of merit $Z T$ can be straightforwardly obtained.

\section{RESULTS AND DISCUSSION}

\section{A. Phonon transport}

We first address the study of the phonon transport properties of the different configurations described in the previous section, namely symmetric, asymmetric, and orthogonal. To
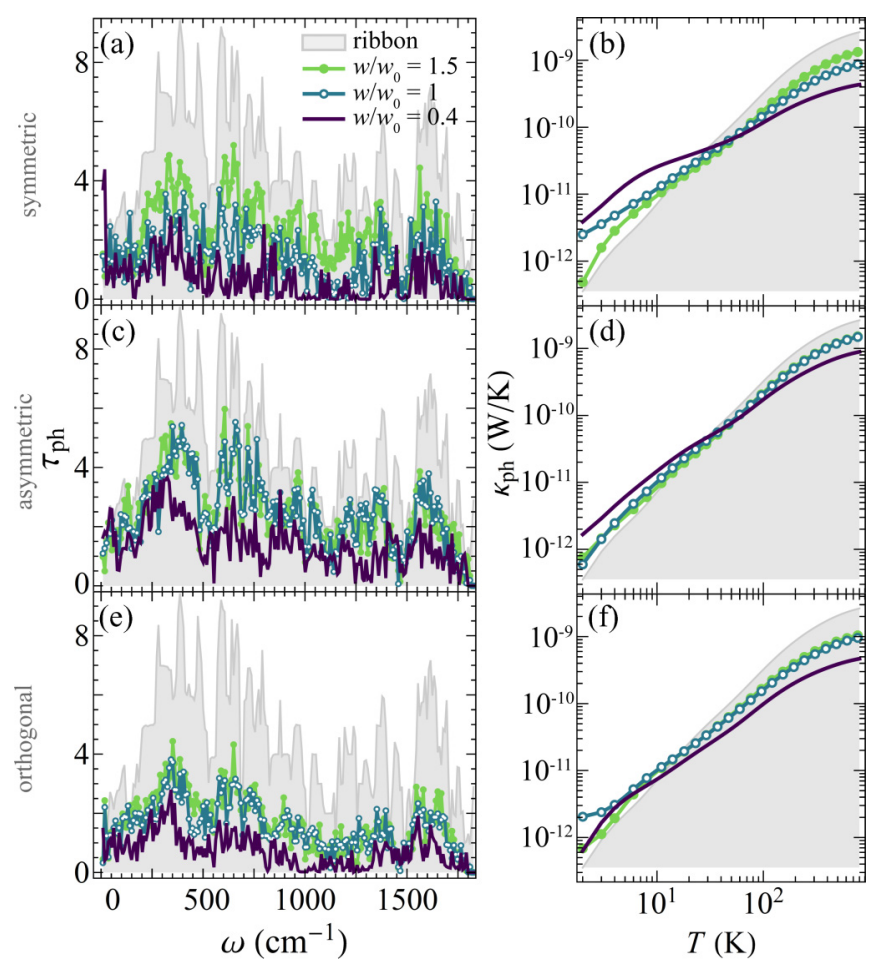

FIG. 2. Phonon transmission $\tau_{\mathrm{ph}}$ as function of the phonon frequency [(a), (c), and (e)] and phonon thermal conductance $\kappa_{\mathrm{ph}}$ as a function of temperature [(b), (d), and (f)] for symmetrical (top), asymmetrical (middle), and orthogonal (bottom) configurations. For all the rings $w_{0}=1.23 \mathrm{~nm}$ and $w$ is indicated in the legend. Gray shadowed curves represent the results for a graphene ribbon of width $w_{0}$.

do so, we keep $w_{0}=1.23 \mathrm{~nm}$ and, for each type of ring, three different arm widths are considered: $w=0.4 w_{0}, w=$ $w_{0}$, and $w=1.5 w_{0}$. Note the latter case does not present a hole.

The phonon transmission coefficient $\tau_{\mathrm{ph}}(\omega)$ and the phonon thermal conductance $\kappa_{\mathrm{ph}}$ for a graphene ribbon of width $w_{0}$ (as reference system) and all the above-mentioned rings are presented in Fig. 2. We observe that there are two clearly distinguishable regimes in the thermal conductance for the three configurations. In the case of symmetric rings, the critical temperature separating both regimes is around 30-50 K [see Fig. 2(b)]. Above this temperature, the ribbon has the highest thermal conductance and for the rings it decreases as the arms becomes narrower. Below that critical temperature, the behavior is radically the opposite and all the rings show a larger conductance than the graphene ribbon, though. In Fig. 2(a) it can also be seen that the phonon transmission $\tau_{\mathrm{ph}}$ decreases for narrower $w$ with the exception of the lowest frequencies, where a peak arises for the case $w=0.4 w_{0}$. Further below, it will be discussed whether or not phonon interference is responsible for this counterintuitive phenomenon.

Both the asymmetric and orthogonal configurations present a similar behavior to the symmetric one. However, while the asymmetric configuration also shows a critical temperature around $30-50 \mathrm{~K}$, the orthogonal one has a lower one around $10 \mathrm{~K}$. Again, below the critical temperature the 

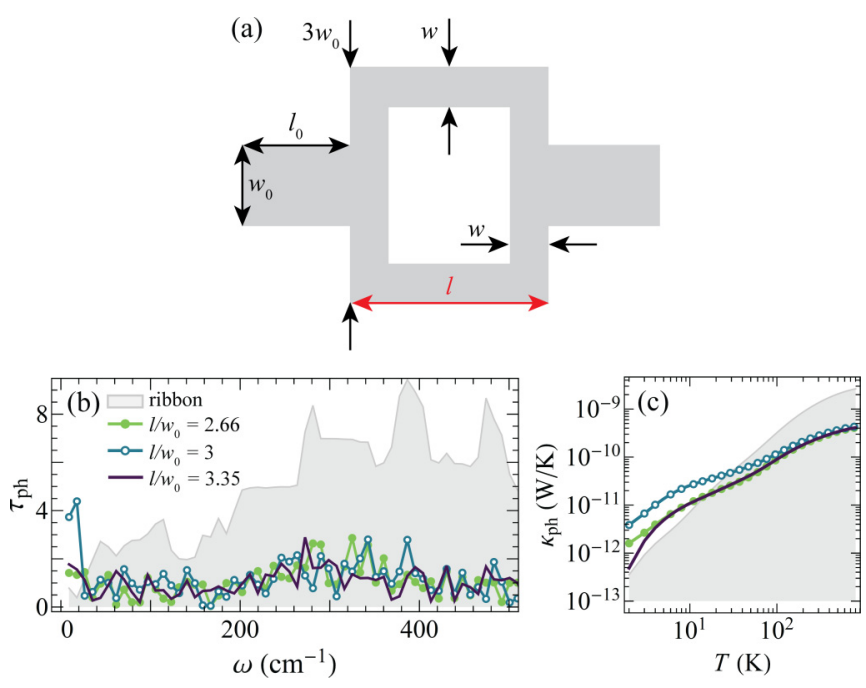

FIG. 3. (a) Schematic diagram of a symmetric ring with $w=$ $0.4 w_{0}$ and varying length $l$. (b) Phonon transmission $\tau_{\mathrm{ph}}$ as function of the phonon frequency and (c) phonon thermal conductance $\kappa_{\mathrm{ph}}$ as a function of temperature for the above depicted rings. For all the rings $w=1.23 \mathrm{~nm}, w=0.4 w_{0}$, and $l$ is indicated in the plot. Gray shadowed curves represent the results for a graphene ribbon of width $w_{0}$.

thermal conductance is higher for the rings when comparing to the graphene ribbon geometry and the role of the inner hole is, a priori, unpredictable. At higher temperatures, the main difference with the symmetric rings is that now the systems with $w=w_{0}$ and $w=1.5 w_{0}$ display almost the same conductance. An explanation of this phenomenon relies on the fact that phonon transport from source to drain is not affected by the hole placed sideways. Once the hole is large enough to alter the transport path, the thermal conductance is degraded.

To assess if the unexpected behavior at low temperatures is due to phonon interference effects, we study the symmetric ring with $w=0.4 w_{0}$ and vary the length of the arms $l$ (see Fig. 3). The transmission for the case $l=3 w_{0}$ was the one that presented a very pronounced peak at low frequencies. However, when we vary $l$, both the peak and the conductance decrease. This could be caused by destructive phonon interference. To further understand it, in Fig. 4 we plot the local phonon density of states (LDOS) at $\omega=18 \mathrm{~cm}^{-1}$, which is the frequency that caused the peak in the transmission spectrum. The LDOS per atomic site is defined as $\eta_{i}(\omega)=$ $-(2 \omega / \pi)\left(\operatorname{Im} G^{r}[\omega]\right)_{i i}$. Accordingly, we observe that for $l=$ $3 w_{0}$ the density is localized in the whole system as it occurs in resonant transmission. When the length is decreased to $l=2.66 w_{0}$ the density is localized at the edges and corners of the ring. However, when $l=3.35 w_{0}$, which was the ring with the lower conductance at low temperature in Fig. 3(c), the phonon density is only localized at the corners. Additionally, in Fig. 4(b) we observe that the dependence of the thermal conductance on the length $l$ is a nonmonotonic function, especially at low temperatures. Similar results were obtained for smaller rings (not shown here). These results reinforced the idea that phonon interference could be responsible for the behavior of graphene rings at low temperatures.
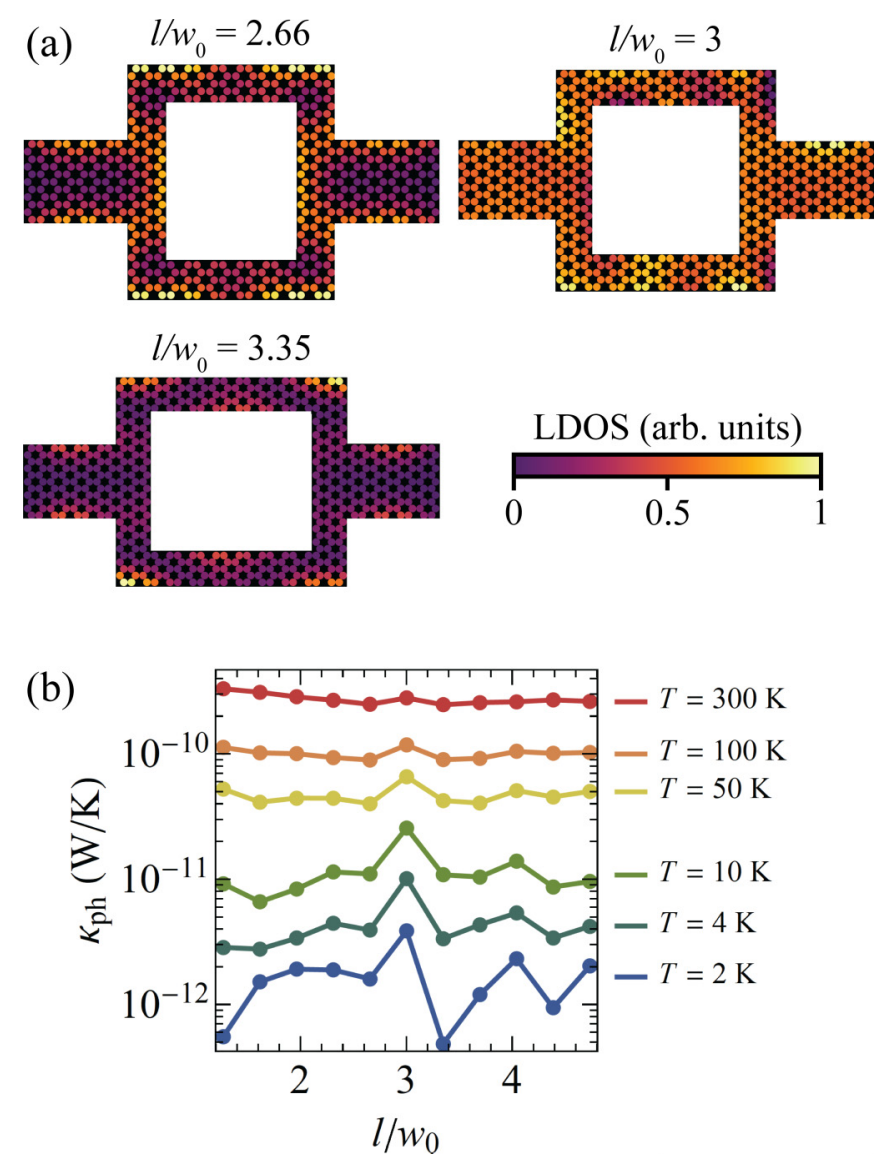

FIG. 4. (a) Local phonon density of states at $\omega=18 \mathrm{~cm}^{-1}$ for the three rings considered in Fig. 3. (b) Phonon thermal conductance $\kappa_{\mathrm{ph}}$ as a function of the length $l$ for several temperatures given in the plot.

We also notice that phonon-phonon interactions should be taken into account at high temperatures. Even though the results are in qualitative good agreement with our previous molecular dynamics study, which include anharmonicities to all orders, where we analyze the thermal conductance of graphene rings with only $w=w_{0}$ [41]. In brief, by using both methods, molecular dynamics and DFTB in combination with Green's functions technique, we see that the thermal conductance of symmetric graphene rings is markedly reduced compared to other configurations. The exception appears at very low temperatures, when quantum effects play a role and classical simulations are no longer valid.

\section{B. Electron transport and thermoelectric response}

Next, we focus on the electron transport through the rings. We set the temperature at $T=4 \mathrm{~K}$ so that quantum interference effects are not smoothed out. The electron transmission $\tau_{\mathrm{el}}$ for all the rings is plotted in the upper panels of Fig. 5. As expected, we observe the symmetric rings present the softer spectrum with smooth resonances, while both the asymmetric and orthogonal show more abrupt features, including Fano resonances (asymmetric line shapes). The latter cases introduce geometrical asymmetries in the system which were shown to induce Fano resonances in the 


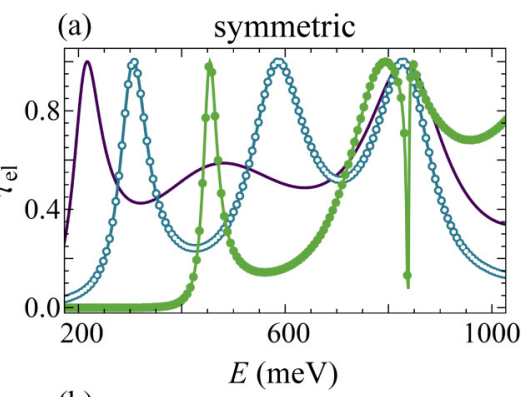

(b)

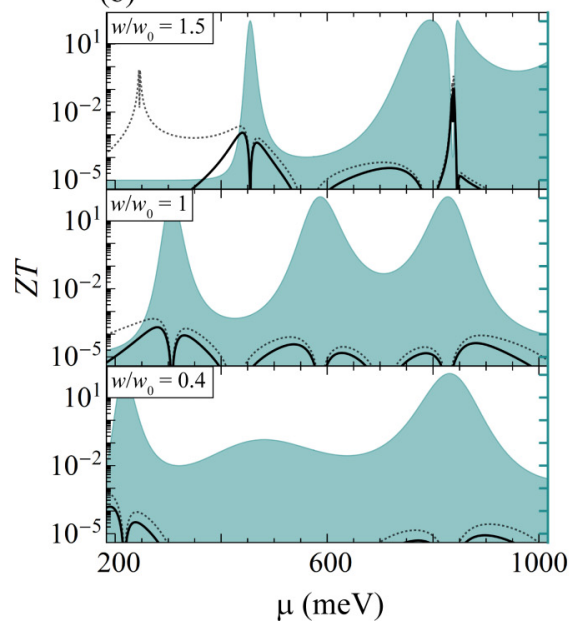

(c)

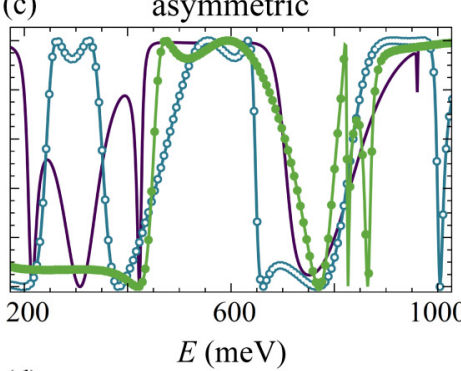

(d)

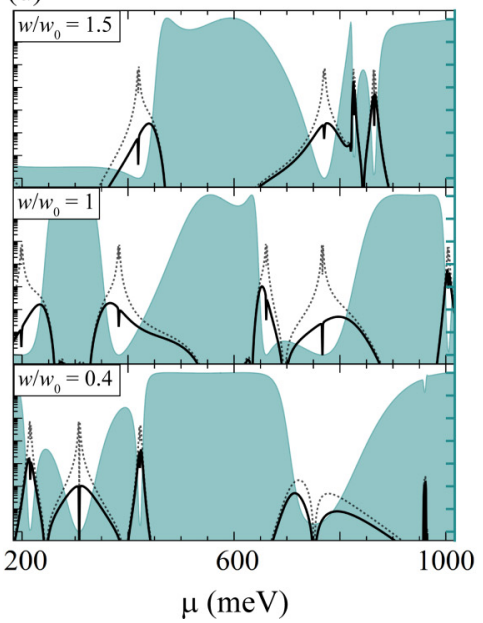

(e)

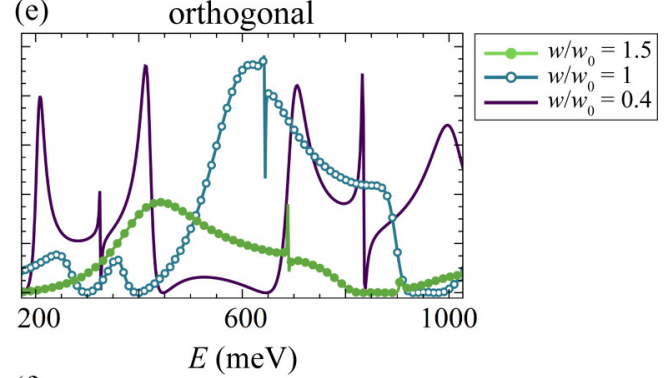

(f)

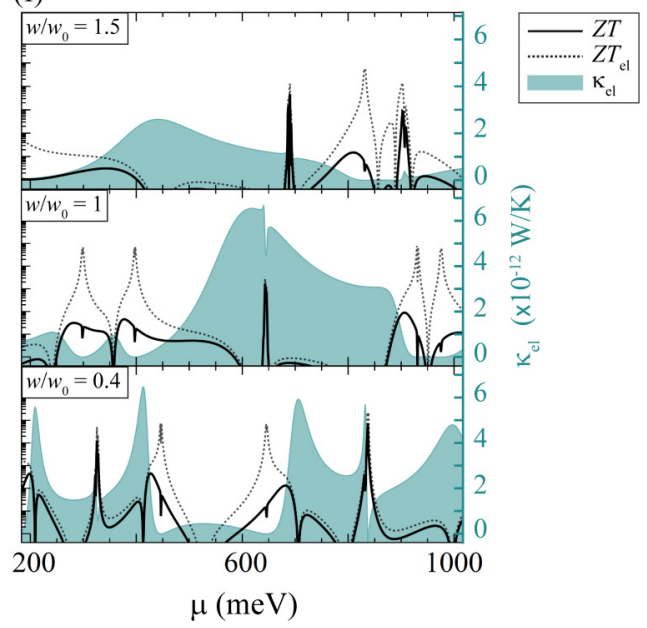

FIG. 5. Electron transmission $\tau_{\mathrm{el}}$ as a function of the Fermi energy for (a) symmetric, (c) asymmetric, and (e) orthogonal rings. Thermoelectric figure of merit $Z T_{\mathrm{el}}$ (electron contribution only) and full $Z T$ (left axis), and electric thermal conductance $\kappa_{\mathrm{el}}$ (right axis) for (b) symmetric, (d) asymmetric, and (f) orthogonal rings.

electron transmission spectrum [40]. The electric thermal conductance $\kappa_{\mathrm{el}}$ is plotted in the lower panels of Fig. 5 along with the electron contribution to the thermoelectric figure of merit $Z T_{\mathrm{el}}=S^{2} \sigma T / \kappa_{\mathrm{el}}$ and the full figure of merit including both electron and phonon contributions, namely $Z T=$ $Z T_{\mathrm{el}} \kappa_{\mathrm{el}} /\left(\kappa_{\mathrm{el}}+\kappa_{\mathrm{ph}}\right)$. In most cases we observe that the order of magnitude of $\kappa_{\mathrm{el}}$ is about $1 \times 10^{-12} \mathrm{~W} / \mathrm{K}$, which is similar to the order of magnitude of the phonon thermal conductance at $4 \mathrm{~K}$. The thermoelectric figure of merit reaches the highest values at energies in the vicinity of Fano resonances and pronounced slopes of the transmission coefficient. In the case of the symmetric configuration, values of $Z T \approx 1$ are only obtained when there is no hole in the system $\left(w=1.5 w_{0}\right)$ since geometries with $w<1.5 w_{0}$ always yield smooth transmission profiles. For the asymmetric and orthogonal rings, values of $Z T$ around unity or even higher are obtained in all cases, indicating that these configurations would be more suitable for thermoelectric applications. As some of the authors demonstrated in Ref. [32], the application of a side-gate voltage between the two arms of the rings would allow us to obtain much higher values of $Z T_{\mathrm{el}}$ for both symmetric and asymmetric configurations. In some cases values as high as 8 were found. The main limitation of that previous study was the exclusion of the phonon contribution to the thermal conductance that ultimately degrades the figure of merit. However, as can be seen in Fig. 5, the addition of the phonon conductance in the calculation of $Z T$ does not significantly degrade the efficiency in the vicinity of abrupt slopes and Fano resonances, which are the features that yield the largest values of $Z T$.

\section{CONCLUSIONS}

In conclusion, we have theoretically studied phonon and electron transport in several graphene nanostructures such as ribbons and rings, to ultimately assess their thermoelectric performance. Regarding the lattice thermal transport, we found that there are two clearly distinguishable regimes in the thermal conductance. For temperatures above the threshold temperature (which lies in the range 10-50 K, depending on the specific geometry configuration) the ribbon presents the highest thermal conductance and, for the rings, it decreases as the hole becomes larger. Also, this hole does not affect thermal transport as long as it does not alter the path from source to drain. These results qualitatively agree with our previous molecular dynamics study [41]. On the contrary, at low temperatures, when semiclassical approaches are no longer valid and quantum effects come into play, the behavior is the opposite since nanorings present higher thermal conductance than the ribbons of the same width in most cases. We suggest, and provide some evidence of phonon interference to be responsible for this counterintuitive behavior, opening new possibilities of engineering phonon transport in a similar way as electron transport in nanorings.

As for electron transport, we found that the asymmetric and the orthogonal configurations show sharper electron 
transmission profiles than the symmetric ones, including Fano resonances. These features give rise to higher values of the thermoelectric figure of merit $Z T$. Precise positions of the Fano features can be highly dependent on the actual system geometry and fabrication imperfections. Nevertheless, as we pointed out in Ref. [32], the use of an additional side gate voltage shifts or may induce Fano resonances, thus allowing for an accurate control of the thermoelectric response by electrostatic means. Additionally, although we observe that electrons and phonons equally contribute to the thermal conductance at $T=4 \mathrm{~K}$, the inclusion of the phonon contribution does not significantly degrade $Z T$ in the vicinity of high slopes and Fano resonances. The low thermal conductance together with the use of quantum interference effects to obtain
Fano resonances to enhance the power factor, make graphene nanorings promising candidates for low temperature thermoelectric applications.

\section{ACKNOWLEDGMENTS}

Work at Madrid was supported by the Agencia Estatal de Investigación of Spain (Grants No. MAT2016-75955 and No. MAT2016-63955). This work has also been partly supported by the German Research Foundation (DFG) within the Cluster of Excellence "Center for Advancing Electronics Dresden." We acknowledge the Center for Information Services and High Performance Computing (ZIH) at TU Dresden for providing computational resources.
[1] H. J. Goldsmid, Introduction to Thermoelectricity (Springer, Berlin, 2010).

[2] L. D. Hicks and M. S. Dresselhaus, Phys. Rev. B 47, 12727 (1993).

[3] L. D. Hicks and M. S. Dresselhaus, Phys. Rev. B 47, 16631 (1993).

[4] G. D. Mahan and J. O. Sofo, Proc. Natl. Acad. Sci. USA 93, 7436 (1996).

[5] G. Gómez-Silva, O. Ávalos-Ovando, M. L. L. de Guevara, and P. A. Orellana, J. Appl. Phys. 111, 053704 (2012).

[6] J. Zheng, M.-J. Zhu, and F. Chi, J. Low Temp. Phys. 166, 208 (2012).

[7] Z.-G. Chen, G. Han, L. Yang, L. Cheng, and J. Zou, Prog. Nat. Sci. Mater. 22, 535 (2012).

[8] V. M. García-Suárez, R. Ferradás, and J. Ferrer, Phys. Rev. B 88, 235417 (2013).

[9] H.-H. Fu, L. Gu, D.-D. Wu, and Z.-Q. Zhang, Phys. Chem. Chem. Phys. 17, 11077 (2015).

[10] M. A. Sierra, M. Saiz-Bretín, F. Domínguez-Adame, and D. Sánchez, Phys. Rev. B 93, 235452 (2016).

[11] R.-N. Wang, G.-Y. Dong, S.-F. Wang, G.-S. Fu, and J.-L. Wang, J. Appl. Phys. 120, 184303 (2016).

[12] A. Khitun, A. Balandin, J. L. Liu, and K. L. Wang, J. Appl. Phys. 88, 696 (2000).

[13] R. Venkatasubramanian, E. Siivola, T. Colpitts, and B. O’Quinn, Nature (London) 413, 597 (2001).

[14] T. C. Harman, P. J. Taylor, M. P. Walsh, and B. E. LaForge, Science 297, 2229 (2002).

[15] A. A. Balandin and O. L. Lazarenkova, Appl. Phys. Lett. 82, 415 (2003).

[16] A. I. Hochbaum, R. Chen, R. D. Delgado, W. Liang, E. C. Garnett, M. Najarian, A. Majumdar, and P. Yan, Nature (London) 451, 163 (2008).

[17] A. I. Boukai, Y. Bunimovich, J. Tahir-Kheli, J.-K. Yu, W. A. Goddard, and J. R. Heath, Nature (London) 451, 168 (2008).

[18] A. V. Savin, Y. S. Kivshar, and B. Hu, Phys. Rev. B 82, 195422 (2010).

[19] J. Hu, S. Schiffli, A. Vallabhaneni, X. Ruan, and Y. P. Chen, Appl. Phys. Lett. 97, 133107 (2010).

[20] Y. Chen, T. Jayasekera, A. Calzolari, K. W. Kim, and M. Buongiorno Nardelli, J. Phys.: Condens. Matter 22, 372202 (2010).
[21] Y. Xu, X. Chen, J.-S. Wang, B.-L. Gu, and W. Duan, Phys. Rev. B 81, 195425 (2010).

[22] F. Mazzamuto, V. Hung Nguyen, Y. Apertet, C. Caër, C. Chassat, J. Saint-Martin, and P. Dollfus, Phys. Rev. B 83, 235426 (2011).

[23] H.-S. Zhang, Z.-X. Guo, X.-G. Gong, and J.-X. Cao, J. Appl. Phys. 112, 123508 (2012).

[24] T. Zhu and E. Ertekin, Phys. Rev. B 90, 195209 (2014).

[25] M. Sharafat Hossain, F. Al-Dirini, F. M. Hossain, and E. Skafidas, Sci. Rep. 5, 11297 (2015).

[26] K.-M. Li, Z.-X. Xie, K.-L. Su, W.-H. Luo, and Y. Zhang, Phys. Lett. A 378, 1383 (2014).

[27] J. E. Barrios-Vargas, B. Mortazavi, A. W. Cummings, R. Martinez-Gordillo, M. Pruneda, L. Colombo, T. Rabczuk, and S. Roche, Nano Lett. 17, 1660 (2017).

[28] J. Haskins, A. Kınacı, C. Sevik, H. Sevinçli, G. Cuniberti, and T. Çağın, ACS Nano 5, 3779 (2011).

[29] F.-P. Du, N.-N. Cao, Y.-F. Zhang, P. Fu, Y.-G. Wu, Z.-D. Lin, R. Shi, A. Amini, and C. Cheng, Sci. Rep. 8, 6441 (2018).

[30] Q. Wu, H. Sadeghi, V. M. García-Suárez, J. Ferrer, and C. J. Lambert, Sci. Rep. 7, 11680 (2017).

[31] Z. Sartipi, A. Hayati, and J. Vahedi, J. Chem. Phys. 149, 114103 (2018).

[32] M. Saiz-Bretín, A. V. Malyshev, P. A. Orellana, and F. Domínguez-Adame, Phys. Rev. B 91, 085431 (2015).

[33] M. Dragoman, A. Dinescu, and D. Dragoman, J. Appl. Phys. 119, 244305 (2016).

[34] Z. Wu, Z. Z. Zhang, K. Chang, and F. M. Peeters, Nanotechnology 21, 185201 (2010).

[35] J. Munárriz, F. Domínguez-Adame, and A. V. Malyshev, Nanotechnology 22, 365201 (2011).

[36] A. Mreńca-Kolasińska and B. Szafran, Phys. Rev. B 94, 195315 (2016).

[37] D. J. P. de Sousa, A. Chaves, J. M. Pereira, and G. A. Farias, J. Appl. Phys. 121, 024302 (2017).

[38] J. Munárriz, F. Domínguez-Adame, P. A. Orellana, and A. V. Malyshev, Nanotechnology 23, 205202 (2012).

[39] R. Farghadan, A. Saffarzadeh, and E. H. Semiromi, J. Appl. Phys. 114, 214314 (2013).

[40] M. Saiz-Bretín, J. Munárriz, A. V. Malyshev, and F. Domínguez-Adame, Phys. Lett. A 379, 2102 (2015). 
[41] M. Saiz-Bretín, A. V. Malyshev, F. Domínguez-Adame, D. Quigley, and R. A. Römer, Carbon 127, 64 (2018).

[42] B. Aradi, B. Hourahine, and T. Frauenheim, J. Phys. Chem. A 111, 5678 (2007).

[43] C. S. Lent and D. J. Kirkner, J. Appl. Phys. 67, 6353 (1990).

[44] D. Z.-Y. Ting, E. T. Yu, and T. C. McGill, Phys. Rev. B 45, 3583 (1992).

[45] J. Schelter, D. Bohr, and B. Trauzettel, Phys. Rev. B 81, 195441 (2010).

[46] H. Sevinçli, C. Sevik, T. Çağin, and G. Cuniberti, Sci. Rep. 3, 1228 (2013).

[47] L. Medrano Sandonas, H. Sevinçli, R. Gutierrez, and G. Cuniberti, Adv. Sci. 5, 1700365 (2018).
[48] T. Niehaus, M. Elstner, T. Frauenheim, and S. Suhai, J. Mol. Struc. 541, 185 (2001).

[49] I. Milošević, N. Kepčija, E. Dobardzić, M. Damnjanović, M. Mohr, J. Maultzsch, and C. Thomsen, Int. J. Mod. Phys. B 24, 655 (2010).

[50] L. Medrano Sandonas, D. Teich, R. Gutierrez, T. Lorenz, A. Pecchia, G. Seifert, and G. Cuniberti, J. Phys. Chem. C 120, 18841 (2016).

[51] L. Medrano Sandonas, R. Gutierrez, A. Pecchia, G. Seifert, and G. Cuniberti, Phys. Chem. Chem. Phys. 19, 1487 (2017).

[52] J. Munárriz, Modelling of Plasmonic and Graphene Nanodevices (Springer, Berlin, 2014). 